\title{
Entropy and growth of expanding periodic orbits for one-dimensional maps
}

by

A. Katok and A. Mezhirov (University Park, Penn.)

Dedicated to the memory of Wiestaw Szlenk

\begin{abstract}
Let $f$ be a continuous map of the circle $S^{1}$ or the interval $I$ into itself, piecewise $C^{1}$, piecewise monotone with finitely many intervals of monotonicity and having positive entropy $h$. For any $\varepsilon>0$ we prove the existence of at least $e^{(h-\varepsilon) n_{k}}$ periodic points of period $n_{k}$ with large derivative along the period, $\left|\left(f^{n_{k}}\right)^{\prime}\right|>e^{(h-\varepsilon) n_{k}}$ for some subsequence $\left\{n_{k}\right\}$ of natural numbers. For a strictly monotone map $f$ without critical points we show the existence of at least $(1-\varepsilon) e^{h n}$ such points.
\end{abstract}

Introduction. This paper is inspired by the seminal work of Michał Misiurewicz and Wiesław Szlenk [MS1, MS2]. For any continuous, piecewise monotone map of the circle or an interval into itself they proved the fundamental inequality

$$
h(f) \leq p(f),
$$

where $h$ is the topological entropy and $p$ is the exponential growth rate of the number of periodic orbits:

$$
p(f)=\limsup _{n \rightarrow \infty} \frac{\log P_{n}(f)}{n},
$$

where $P_{n}(f)=\operatorname{Card}\left\{x: f^{n} x=x\right\}$.

Our main goal is to show that for smooth or piecewise smooth maps a large number of periodic orbits are expanding with exponent at least almost as large as entropy.

TheOREM 1. Let $f: S^{1} \rightarrow S^{1}$ be a $C^{1}$ monotone map without critical points with $|\operatorname{deg} f|=k, k>1$ (hence $h(f)=\log k)$. Then for each $\varepsilon>0$

1991 Mathematics Subject Classification: Primary 58F.

Research of the first author partially supported by NSF grant DMS-97-04776. 
and $n$ large enough one can find at least $(1-\varepsilon) k^{n}$ periodic points $x_{i}^{n}$ of period $n$ for which $\left|\left(f^{n}\right)^{\prime}\left(x_{i}^{n}\right)\right| \geq(k-\varepsilon)^{n}$.

TheOREM 2. Let $f$ be a continuous, piecewise $C^{1}$ map of $S^{1}$ or I into itself with finitely many critical points and with entropy $h(f)=h>0$. Then for each $\varepsilon>0$ one can find a subsequence $\left\{n_{k}\right\}_{k=0}^{\infty}$ of natural numbers such that for each $n_{k}$ the function $f$ has at least $e^{(h-\varepsilon) n_{k}}$ periodic points $x_{i}^{n_{k}}$ of period $n_{k}$ for which $\left|\left(f^{n_{k}}\right)^{\prime}\left(x_{i}^{n_{k}}\right)\right|>e^{(h-\varepsilon) n_{k}}$.

In both statements the loss of expansion is small on the exponential scale. The loss in the number of periodic points is small in the stronger multiplicative sense for monotone maps of the circle but only in the exponential sense if critical points are present. This exponential loss in the number of points with large derivative is probably unavoidable even if we assume higher smoothness.

One can naturally ask whether for monotone maps of the circle one can actually guarantee that no expansion is lost. An easy answer is that the expansion is actually controlled not by entropy but by the Lyapunov exponent with respect to the maximal entropy measure which is always greater than or equal to the entropy; if the inequality is strict, which is generically true, one can avoid the loss of expansion altogether (Proposition 4). We discuss the difficulties of the remaining case at the end of the paper.

Our results can be viewed as a simple model case for the still unknown $C^{1}$ versions of results connecting entropy and the growth of periodic orbits for $C^{1+\varepsilon}$ diffeomorphisms in dimension two and flows in dimension three, specifically [K1, Theorem 4.3; K2, Theorem 4.1; KM, Corollary S.5.11]. For the proofs of those results the $C^{1+\varepsilon}$ assumption is crucial since they heavily rely on Pesin theory [P, KM, BKP], essential elements of which fail in the $C^{1}$ case $[\mathrm{Pu}]$.

The novelty of our approach lies in not requiring any regularity beyond $C^{1}$ while the influence of critical points is overcome in a more or less standard fashion by considering a Markov approximation for orbits avoiding a neighborhood of the critical set. If we assume that $f$ is $C^{1+\varepsilon}$ for some positive $\varepsilon$ then Theorem 1 becomes a simpler version of the results for the two-dimensional invertible case (e.g. [KM, Corollary S.5.11]) and Theorem 2 can be deduced from those results via the Markov approximation (see proof of Theorem 2 below). The argument in the $C^{1+\varepsilon}$ case uses existence of orbits regular with respect to a measure with high entropy which return very close to the initial condition and then one uses a non-uniform version of the Anosov closing lemma for hyperbolic systems (see [KM, Theorem S.4.13]). The latter depends on a bounded distortion estimate which is exactly where Hölder regularity of the derivative is required. Uniform bounded distortion breaks down for $C^{1}$ maps so we have to replace it with a counting argument 
showing that it still holds in many places. While completely elementary, the argument is "ergodic" in spirit and possibly its more sophisticated versions may be used in the two-dimensional case.

We would like to thank the referees for their comments which helped to improve the presentation of the paper. In particular, we are grateful for pointing out the argument and references which produce a multiplicative lower bound for the number of periodic points (see subsection "Multiplicative growth estimate" below).

Proofs. Theorems 1 and 2 will be deduced from the following result.

Theorem 3. Let $f$ be a continuous, piecewise $C^{1}$ map of $S^{1}$ or I into itself. Assume that there exist $a>1, M>0$ and $\beta>0$ and an increasing sequence $\left\{n_{l}\right\}$ of positive integers such that for each $n_{l}$ one can find $\left[a^{n_{l}}\right]$ non-intersecting intervals $\left\{\Delta_{i}^{n_{l}}\right\}_{i=1}^{\left[a^{n_{l}}\right]}$ in $S^{1}$ with the following properties:

(1) $f$ is $C^{1}$ on each interval $f^{m}\left(\Delta_{i}^{n_{l}}\right)$ for $m=0,1, \ldots, n_{l}-1$,

(2) each $\Delta_{i}^{n_{l}}$ contains a periodic point $x_{i}^{n_{l}} \in \Delta_{i}^{n_{l}}$ of period $n_{l}$,

(3) the derivative of $f$ at each $x_{i}^{n_{l}}$ and at each of its images is greater than $\beta$ in absolute value,

(4) $\quad \sum_{m=0}^{n_{l}-1} \sum_{i=1}^{\left[a^{n_{l}}\right]} l\left(f^{m}\left(\Delta_{i}^{n_{l}}\right)\right) \leq M a^{n_{l}}$, where $l(\Delta)$ is the length of the interval $\Delta$,

(5) $\quad l\left(f^{n_{l}}\left(\Delta_{i}^{n_{l}}\right)\right) \geq \beta$.

Then for each $\varepsilon>0$ one can find $N \in \mathbb{N}$ such that for each $n_{l}>N$ the function $f$ has at least $(1-\varepsilon) a^{n_{l}}$ periodic points $x_{i}$ of period $n_{l}$ with $\left|\left(f^{n_{l}}\right)^{\prime}\left(x_{i}\right)\right|>(a-\varepsilon)^{n_{l}}$.

Condition (5) will give us the existence in most $\Delta_{i}^{n_{l}}$ 's of a point $y_{i}^{n_{l}}$ where the function $f^{n_{l}}$ has large derivative, $\left|\left(f^{n_{l}}\right)^{\prime}\left(y_{i}^{n_{l}}\right)\right|>(a-\varepsilon)^{n_{l}}$. Condition (2) will guarantee the existence of a periodic point $x_{i}^{n_{l}}$ of period $n_{l}$ in each interval $\Delta_{i}^{n_{l}}$ containing $y_{i}^{n_{l}}$, and conditions (1), (3) and (4) will give us the estimate

$$
\left|\frac{\left(f^{n_{l}}\right)^{\prime}\left(y_{i}^{n_{l}}\right)}{\left(f^{n_{l}}\right)^{\prime}\left(x_{i}^{n_{l}}\right)}\right|<e^{\varepsilon n_{l}}
$$

for most intervals $\Delta_{i}^{n_{l}}$.

Denote by $\omega_{g}(\delta)$ the $\delta$-modulus of continuity of a function $g$,

$$
\omega_{g}(\delta)=\sup \{|g(x)-g(y)||| x-y \mid<\delta\} .
$$

In the proof of Theorem 3 we use the following lemma. 
LEMma 1. Let $f$ be a $C^{1}$ map of the circle or an interval into itself and $\psi: \mathbb{N} \rightarrow \mathbb{R}_{+}$be a function with $\psi(n)=o(n)$. For each natural $n$ define

$$
\phi(n)=\phi_{f, \psi}(n)=\sup \left\{\sum_{i=1}^{n} \omega_{f^{\prime}}\left(\delta_{i}\right) \mid \sum_{i=1}^{n} \delta_{i}<\psi(n), 0 \leq \delta_{i} \leq 1\right\} .
$$

Then $\phi(n)=o(n)$.

Proof. Fix $\varepsilon>0$. We show that there exists $N$ such that $\phi(n) \leq \varepsilon n$ for each $n>N$. As $f^{\prime}$ is uniformly continuous, $\lim _{\delta \rightarrow 0} \omega_{f^{\prime}}(\delta)=0$, so there exists $\gamma$ such that $\omega_{f^{\prime}}(\delta)<\varepsilon / 2$ if $\delta<\gamma$. Then if $\sum_{i=1}^{n} \delta_{i} \leq \psi(n)$ we have $\operatorname{Card}\left(\left\{i \mid \delta_{i} \geq \gamma\right\}\right)<\psi(n) / \gamma$, and hence

$$
\sum_{i=1}^{n} \omega_{f^{\prime}}\left(\delta_{i}\right)=\sum_{i: \delta_{i}<\gamma}+\sum_{i: \delta_{i} \geq \gamma} \leq \frac{\varepsilon}{2} n+\frac{\psi(n)}{\gamma} \omega_{f^{\prime}}(1) \leq \varepsilon n
$$

for $n$ large enough, as $\psi(n)=o(n)$.

Proof of Theorem 3. Fix $\varepsilon>0$. For each $n_{l}$ we have $A=\left[a^{n_{l}}\right]$ nonintersecting intervals $\left\{\Delta_{i}^{n_{l}}\right\}_{i=1}^{A}$, so the sum of their lengths $\sum_{i=1}^{A} l\left(\Delta_{i}^{n_{l}}\right)$ is at most 1 . Hence all but at most $(a-\varepsilon)^{n_{l}}$ intervals have length smaller than $(a-\varepsilon)^{-n_{l}}$. Furthermore, for any interval, $l\left(f^{n_{l}}\left(\Delta_{i}^{n_{l}}\right)\right) \geq \beta$, so in each of those $A-(a-\varepsilon)^{n_{l}}$ intervals there exists a point $y_{i}^{n_{l}} \in \Delta_{i}^{n_{l}}$ with

$$
\left|\left(f^{n_{l}}\right)^{\prime}\left(y_{i}^{n_{l}}\right)\right| \geq \frac{\beta}{(a-\varepsilon)^{-n_{l}}}=\beta(a-\varepsilon)^{n_{l}} .
$$

As $\sum_{i=1}^{A} \sum_{k=0}^{n_{l}-1} l\left(f^{k}\left(\Delta_{i}^{n_{l}}\right)\right) \leq M a^{n_{l}}$, we know that

$$
\operatorname{Card}\left(\left\{i \mid \sum_{k=0}^{n_{l}-1} l\left(f^{k}\left(\Delta_{i}^{n_{l}}\right)\right) \geq \sqrt{n_{l}}\right\}\right) \leq a^{n_{l}} \frac{M}{\sqrt{n_{l}}} .
$$

So, at least $A-a^{n_{l}} M / \sqrt{n_{l}}-(a-\varepsilon)^{n_{l}}$ intervals $\Delta_{i}^{n_{l}}$ contain a point $y_{i}^{n_{l}}$ in their interior and satisfy the inequality

$$
\sum_{k=0}^{n_{l}-1} l\left(f^{k}\left(\Delta_{i}^{n_{l}}\right)\right) \leq \sqrt{n_{l}}
$$

Let $x_{i}^{n_{l}}$ be our periodic point in one of those intervals. Let $S_{i}$ be the set of integers $k$ between 0 and $n_{l}-1$ such that

$$
\left|\left(f^{n_{l}}\right)^{\prime}\left(f^{k}\left(x_{i}^{n_{l}}\right)\right)\right| \leq\left|\left(f^{n_{l}}\right)^{\prime}\left(f^{k}\left(y_{i}^{n_{l}}\right)\right)\right| .
$$

Then we have

$$
\begin{aligned}
\log \left|\frac{\left(f^{n_{l}}\right)^{\prime}\left(y_{i}^{n_{l}}\right)}{\left(f^{n_{l}}\right)^{\prime}\left(x_{i}^{n_{l}}\right)}\right| & \leq \sum_{k \in S_{i}}\left(\log \left|f^{\prime}\left(f^{k}\left(y_{i}^{n_{l}}\right)\right)\right|-\log \left|f^{\prime}\left(f^{k}\left(x_{i}^{n_{l}}\right)\right)\right|\right) \\
& \leq \beta^{-1} \sum_{k \in S_{i}}\left(\left|f^{\prime}\left(f^{k}\left(y_{i}^{n_{l}}\right)\right)\right|-\left|f^{\prime}\left(f^{k}\left(x_{i}^{n_{l}}\right)\right)\right|\right)
\end{aligned}
$$




$$
\begin{aligned}
& \leq \beta^{-1} \sum_{k=0}^{n_{l}-1}\left\|f^{\prime}\left(f^{k}\left(y_{i}^{n_{l}}\right)\right)|-| f^{\prime}\left(f^{k}\left(x_{i}^{n_{l}}\right)\right)\right\| \\
& \leq \beta^{-1} \sum_{k=0}^{n_{l}-1} \omega_{f^{\prime}}\left(l\left(f^{k}\left(\Delta_{i}^{n_{l}}\right)\right)\right) .
\end{aligned}
$$

Here we assume that the logarithms are natural.

By Lemma 1 we know that if $n_{l}>N_{0}$ then $\sum_{k=0}^{n-1} \omega_{f^{\prime}}\left(l\left(f^{k}\left(\Delta_{i}^{n_{l}}\right)\right)\right)<$ $\varepsilon \beta n_{l}$, hence for all $n_{l}$ large enough we have

$$
\log \left|\frac{\left(f^{n_{l}}\right)^{\prime}\left(y_{i}^{n_{l}}\right)}{\left(f^{n_{l}}\right)^{\prime}\left(x_{i}^{n_{l}}\right)}\right| \leq \varepsilon n_{l} .
$$

From this we get

$$
\left|\left(f^{n_{l}}\right)^{\prime}\left(x_{i}^{n_{l}}\right)\right| \geq \frac{\left|\left(f^{n_{l}}\right)^{\prime}\left(y_{i}^{n_{l}}\right)\right|}{e^{\varepsilon n_{l}}} \geq \frac{(a-\varepsilon)^{n_{l}}}{e^{\varepsilon n_{l}}}=\left(\frac{a-\varepsilon}{e^{\varepsilon}}\right)^{n_{l}}
$$

for all $n_{l}$ large enough, which completes the proof.

We will use the following remarks in the proof of Theorem 1 , Theorem 2 and Proposition 4.

REMARK 1. Suppose that instead of condition (5) the intervals $\Delta_{i}^{n_{l}}$ satisfy the following condition:

(5a) There exists $b>0$ such that for all $\gamma>0$ and for $n_{l}$ large enough, in at least $(1-\gamma) a^{n_{l}}$ intervals $\Delta_{i}^{n_{l}}$ one can find a point $y_{i}^{n_{l}}$ such that

$$
\left|\left(f^{n_{l}}\right)^{\prime}\left(y_{i}^{n_{l}}\right)\right| \geq b^{n_{l}} .
$$

Then $f$ has at least $(1-\varepsilon) a^{n_{l}}$ periodic points $x_{i}^{n_{l}}$ of period $n_{l}$ with

$$
\left|\left(f^{n_{l}}\right)^{\prime}\left(x_{i}^{n_{l}}\right)\right| \geq(b-\varepsilon)^{n_{l}} .
$$

REMARK 2. If conditions (1)-(5) hold for all $n$ large enough, say $n>N$, the conclusion will hold in the sense of existence of periodic points of all periods $n>N$.

Proof of Theorem 1. Let $f: S^{1} \rightarrow S^{1}$ be a monotone $C^{1}$ map of degree $k,|k|>1$, without critical points. To prove the theorem we check that conditions (1)-(5) of Theorem 3 are satisfied for $a=k$. As $f$ is smooth on the whole circle condition (1) is true.

For each positive integer $n$ take a standard decomposition of the circle into $k^{n}$ intervals $\left\{\Delta_{i}^{n}\right\}_{i=1}^{k^{n}}$, each mapped by $f^{n}$ onto the whole $S^{1}$ and hence containing a periodic point of $f$ of period $n$. For example, pre-images of a fixed point of order up to $n$ may serve as endpoints of these intervals. As $f$ does not have critical points its derivative is bounded away from zero on the whole circle. Condition (3) then holds with any $\beta<\min \left|f^{\prime}\right|$. 
As for each $i$ and each positive $n$ one has $f^{n}\left(\Delta_{i}^{n}\right)=S^{1}$, to satisfy (5) we can take any $\beta<1$. Further, for each $m$ the intervals $\left\{f^{m}\left(\Delta_{i}^{n}\right)\right\}_{i=1}^{k^{n}}$ cover the circle exactly $k^{m}$-fold, which implies

$$
\sum_{i=1}^{k^{n}} l\left(f^{m}\left(\Delta_{i}^{n}\right)\right)=k^{m} \quad \text { and } \quad \sum_{m=0}^{n-1} \sum_{i=1}^{k^{n}} l\left(f^{m}\left(\Delta_{i}^{n}\right)\right) \leq k^{n} .
$$

Now conditions (1)-(5) of Theorem 3 are satisfied for $a=k$ and $M=1$ from which it follows that for each $\varepsilon>0$ and all $n$ large enough there are at least $(1-\varepsilon) k^{n}$ periodic points $x_{i}^{n}$ of period $n$ with $\left|\left(f^{n}\right)^{\prime}\left(x_{i}^{n}\right)\right|>(k-\varepsilon)^{n}$.

To prove Theorem 2 we use [KH, Corollary 15.2.9] which says that the topological entropy of a continuous piecewise monotone map $f$ of $I$ or $S^{1}$ into itself can be approximated arbitrarily well by the topological entropy of Markov chains for iterates of $f$ associated with collections of subintervals.

Proof of Theorem 2. We call a point $x$ singular for the function $f$ if either $f$ is not $C^{1}$ in a neighborhood of $x$ or $f^{\prime}(x)=0$. Singular points divide the domain of $f$ into finitely many intervals such that on each interval the derivative is continuous and strictly positive in the interior. Fix an $\varepsilon>0$. By [KH, Corollary 15.2.9] one can construct a one-sided topological Markov chain $\left(\Omega_{A}^{R}, \sigma_{A}^{R}=\sigma_{\uparrow \Omega_{A}^{R}}\right)$ for an iterate of $f$ with entropy $h\left(\sigma_{\uparrow \Omega_{A}^{R}}\right)>h-\varepsilon$. We still denote this iterate by $f$ since it satisfies the assumptions of the theorem. Following $[\mathrm{KH}]$ we use the upper index $R$ for one-sided topological Markov chains to distinguish them from two-sided ones. To apply Theorem 3 we consider an invariant subset of $\Omega_{A}^{R}$ such that the subintervals corresponding to periodic points in $\Omega_{A}^{R}$ do not contain singular points of $f$.

The function $f$ can only have finitely many singular points and those which belong to $\Omega_{A}^{R}$ can be coded by sequences

$$
\omega^{(i)}=\left(\omega_{1}^{i}, \omega_{2}^{i}, \ldots, \omega_{n}^{i}, \ldots\right) \in \Omega_{A}^{R}, \quad i=1, \ldots, q .
$$

For a positive integer $r$ consider the subset $F=F(r)$ of $\Omega_{A}^{R}$ that consists of all elements of $\Omega_{A}^{R}$ which contain no finite subsequence $\left(\omega_{1}^{i}, \omega_{2}^{i}, \ldots, \omega_{r}^{i}\right)$ for $i=1, \ldots, q$. Obviously, $F$ is $\sigma$-invariant for any value of $r$. As there are only finitely many sequences $\omega^{(i)}$ we can take $r$ large enough that $h\left(\sigma_{\uparrow F}\right)>$ $h_{\Omega_{A}^{R}}-\varepsilon>h-2 \varepsilon$. (See [Li], where also an exponential estimate from above for the drop in entropy is obtained.)

Notice that once we fix $r$, by the construction of $F$ we can find $\delta>0$ such that all points corresponding to sequences in $F$ lie outside the $\delta$-neighborhood of the set of singular points. In fact, otherwise for any positive $\delta$ we could find a point $x$ and the corresponding sequence $\omega=\left(\omega_{1}, \omega_{2}, \ldots, \omega_{n}, \ldots\right)$ such that some iterate $f^{s}(x)$ would belong to the $\delta$-neighborhood of some singular point. For sufficiently small $\delta$ this would imply that $\omega^{(i)}=\left(\omega_{1}^{i}, \omega_{2}^{i}, \ldots, \omega_{r}^{i}, \ldots\right)$ and $\sigma^{s} \omega=\left(\omega_{s+1}, \omega_{s+2}, \ldots, \omega_{s+r}, \ldots\right)$ 
have at least $r$ first elements equal: $\omega_{s+1}=\omega_{1}^{i}, \ldots, \omega_{s+r}=\omega_{r}^{i}$. But it would mean that $\omega$ contains the subsequence $\left(\omega_{1}^{i}, \omega_{2}^{i}, \ldots, \omega_{r}^{i}\right)$ and hence cannot belong to $F$.

From this we see that the derivative of $f$ is uniformly bounded away from 0 at all the periodic points of $f$ with corresponding sequences in $F$.

By the choice of $F, h\left(\sigma_{\uparrow F}\right)>h-2 \varepsilon$ and we can find an increasing sequence $\left\{n_{k}\right\}_{k=1}^{\infty}$ of positive integers such that $F$ contains at least $e^{\left(h\left(\sigma_{\mid F}\right)-\varepsilon\right) n_{k}}>e^{(h-3 \varepsilon) n_{k}}$ different periodic sequences of period $n_{k}$. The subintervals $\Delta_{i}^{\left(n_{k}\right)}$ corresponding to the first $n_{k}$ elements of those periodic sequences satisfy the conditions of Theorem 3 for $a=e^{h-3 \varepsilon}$ and so give us at least $\frac{1}{2} e^{(h-3 \varepsilon) n_{k}}$ periodic points $x_{i}^{n_{k}}$ of period $n_{k}$ with $\left|f^{\prime}\left(x_{i}^{n_{k}}\right)\right|>e^{(h-4 \varepsilon) n_{k}}$.

REMARK 3. With a slight modification the above proof works for maps whose set of critical points consists of finitely many isolated points and intervals.

The next proposition shows that if in Theorem 1 the exponent $\chi$ of the measure $\mu$ of maximal entropy for $f$ is strictly greater than $\log k$ then one can get a stronger estimate for the derivative of $f$ along periodic orbits.

Proposition 4. Let $f: S^{1} \rightarrow S^{1}$ be a $C^{1}$ monotone map without critical points with $|\operatorname{deg} f|=k>1$. Let $\tau=e^{\chi}$ be strictly greater than $k, \tau=k+\alpha$, $\alpha>0$. Then for each $\varepsilon>0$ and all $n$ large enough one can find at least $(1-\varepsilon) k^{n}$ periodic points $x_{i}^{n}$ of period $n$ for which $\left|\left(f^{n}\right)^{\prime}\left(x_{i}^{n}\right)\right|>k^{n}$.

Pr o of. To prove the proposition we check that condition (5a) of Theorem 3 is satisfied for $a=k$ and $b=k+\frac{1}{2} \alpha>k$.

As $f$ does not have critical points the function $\varphi=\log f^{\prime}$ is bounded and hence integrable. As $\mu$ is ergodic the Birkhoff Ergodic Theorem tells us that $n^{-1} \sum_{k=0}^{n-1} \varphi\left(f^{k}(x)\right)=n^{-1} \log \left(f^{n}\right)^{\prime}(x)$ converges to the constant $\chi=\log \tau$ $\mu$-almost everywhere. This implies that as $n \rightarrow \infty$,

$$
\mu\left(\left\{x \mid\left(f^{n}\right)^{\prime}(x)<\left(\tau-\frac{1}{2} \alpha\right)^{n}\right\}\right) \rightarrow 0 .
$$

But as $\mu$ is a counting measure on the intervals $\Delta_{i}^{n}$ we get

$$
\text { Card }\left\{i \mid\left(f^{n}\right)^{\prime}(x)<\left(\tau-\frac{1}{2} \alpha\right)^{n} \forall x \in \Delta_{i}^{n}\right\}=o\left(k^{n}\right),
$$

which gives us the condition (5a) for $b=\tau-\frac{1}{2} \alpha=k+\frac{1}{2} \alpha>k$.

As the function $f$ satisfies the conditions of Theorem 1 it also satisfies conditions (1)-(4) of Theorem 3, which proves the proposition.

\section{Open questions and comments}

Infinite critical sets. The inequality

$$
h(f) \leq p(f)
$$


established by Misiurewicz and Szlenk for piecewise monotone continuous maps of the circle or an interval has been extended by Misiurewicz to arbitrary continuous maps [M1, ALM]. Correspondingly, it is natural to ask whether our results about abundance of fast expanding periodic points also hold for arbitrary $C^{1}$ or continuous piecewise $C^{1}$ maps. In order to carry out the scheme of our proof we need to know that the invariant set of points which avoid a sufficiently small neighborhood of the critical set carries topological entropy which converges to the topological entropy of the map as the size of the neighborhood decreases. M. Misiurewicz pointed out to an argument in his paper [M2] which, while dealing with piecewise monotone maps, may be useful in establishing this fact.

Multiplicative expansion estimate. Another question is whether in the statement of Theorem 1 one can replace the inequality $\left|\left(f^{n}\right)^{\prime}\left(x_{i}^{n}\right)\right| \geq(k-\varepsilon)^{n}$ by a stronger inequality: either $\left|\left(f^{n}\right)^{\prime}\left(x_{i}^{n}\right)\right| \geq k^{n}$ or at least $\left|\left(f^{n}\right)^{\prime}\left(x_{i}^{n}\right)\right| \geq$ $C k^{n}$ for a positive constant $C$. One needs to consider only the case not covered by Proposition 4, namely a $C^{1}$ monotone map $f: S^{1} \rightarrow S^{1}$ with $|\operatorname{deg} f|=k>1$ without critical points such that $\tau=e^{\chi}=k$. Even in the expanding case $\left|f^{\prime}\right|>1$ the situation is not completely clear. If $f$ is a $C^{1+\varepsilon}$ expanding map for some $\varepsilon>0$ the situation is rigid: the maximal entropy measure is absolutely continuous, in fact, it has a continuous positive density and the conjugacy with the linear expanding map $E_{k}$ is smooth. Hence, for all points of period $n$ the derivative of $f^{n}$ is exactly $k^{n}$. However, A. Quas [Q] has shown that the rigidity result does not extend to $C^{1}$ maps. While in his examples the periodic points are not affected (the derivatives are still equal to exactly $k^{n}$ ) this makes an improvement of Theorem 1 mentioned above rather unlikely. The main reason the usual argument establishing rigidity fails is the loss of the uniform bounded distortion estimate which is also responsible for examples of $C^{1}$ expanding maps without an absolutely continuous invariant measure [GS] and for a similar pathology such as "thick" invariant Cantor sets [Ho, Theorem 4].

Two-dimensional case. As mentioned in the introduction, one of our motivations was to try to find a method which may lead to a proof of the Misiurewicz-Szlenk inequality $h(f) \leq p(f)$ for any $C^{1}$ diffeomorphism of a compact two-dimensional manifold. In fact, a proper conjecture would be the following "semilocal" statement:

Let $M$ be a two-dimensional manifold, $U \subset M$ its open subset and $\Lambda \subset U$ be a compact set. If $f: U \rightarrow M$ is a $C^{1}$ diffeomorphic embedding such that $f \Lambda=\Lambda$ then for any open neighborhood $V \supset \Lambda$,

$$
h\left(f_{\uparrow \Lambda}\right) \leq p\left(f_{\uparrow V}\right) .
$$


Multiplicative growth estimate. For piecewise monotone maps the Misiurewicz-Szlenk inequality can be strengthened to a multiplicative estimate for the growth of the number of periodic orbits:

$$
\limsup _{n \rightarrow \infty} \frac{P_{n}(f)}{\exp n h(f)}>0 .
$$

While we have not found an explicit reference to that result in the literature it can be easilly deduced from the Milnor-Thurston kneading theory $[\mathrm{MlT}]$ and the work of Hofbauer [Ho]. This reduction was suggested by one of the referees. It works as follows. By $[\mathrm{MlT}]$ a piecewise monotone interval map with positive entropy is semiconjugate to a map $g$ with the same entropy and a constant absolute value of the slope. This semiconjugacy is non-decreasing, so the fiber over a periodic point of $g$ is a point or an interval, and therefore it contains a periodic point of the original map. Thus, it is enough to prove the estimate for $g$. But such a map $g$ has an absolutely continuous ergodic measure $\mu$ which is supported on finitely many intervals (this follows, for example, from [LY]). The entropy $h_{\mu}(g)$ of $g$ with respect to this measure must be equal to the Lyapunov exponent which is equal to the absolute value of the slope and hence by Ruelle's entropy inequality, $h_{\mu}(g)=h(g)$. Denote the support of $\mu$ by $J$. Thus $h(g)=h\left(g_{\lceil J}\right)$ and since $g$ is topologically transitive on $J$ by [Ho, Theorem 3], the inequality $(*)$ holds.

Using a further development of the Hofbauer technique J. Buzzi $[\mathrm{Bu}$, Theorems 1.1 and 2.9] proved existence and structural results for arbitrary $C^{\infty}$ maps of the circle or an interval which in particular imply the multiplicative estimate $(*)$. He also shows $[\mathrm{Bu}$, Appendix $\mathrm{A}]$ that $(*)$ does not hold for $C^{r}$ maps for any finite $r$.

On the other hand, such estimates are known for certain classes of dynamical systems in dimensions higher than one. In particular, they appear in systems with uniformly hyperbolic behavior, such as Axiom A diffeomorphisms or locally maximal hyperbolic sets (see e.g. [KH, Theorem 18.5.5]). There are other situations where such estimates are known, the most remarkable being $C^{\infty}$ diffeomorphisms of compact two-dimensional manifolds $[\mathrm{KT}]$ and also geodesic flows on rank one manifolds of non-positive curvature $[\mathrm{Kn}]$.

\section{References}

[ALM] L. Alsedà, J. Llibre and M. Misiurewicz, Combinatorial Dynamics and Entropy in Dimension One, World Sci., Singapore, 1993.

[BKP] L. Barreira, A. Katok and Ya. Pesin, Non-Uniformly Hyperbolic Dynamical Systems, monograph in preparation.

[Bu] J. Buzzi, Intrinsic ergodicity of smooth interval maps, Israel J. Math. 100 (1997), $125-161$. 
[GS] P. Góra et B. Schmitt, Un exemple de transformation dilatante et $C^{1}$ par morceaux de l'intervalle, sans probabilité absolument continue invariante, Ergodic Theory Dynam. Systems 9 (1989), 101-113.

[Ho] F. Hofbauer, The structure of piecewise monotonic transformations, ibid. 1 (1981), 159-178.

[K1] A. Katok, Lyapunov exponents, entropy and periodic points for diffeomorphisms, Publ. Math. IHES 51 (1980), 137-173.

[K2] - Entropy and closed geodesics, Ergodic Theory Dynam. Systems 2 (1982), 339-367.

[KH] A. Katok and B. Hasselblatt, Introduction to the Modern Theory of Dynamical Systems, Cambridge Univ. Press, New York, 1995.

[KM] A. Katok and L. Mendoza, Dynamical systems with non-uniformly hyperbolic structure, supplement to [KH], 659-700.

[Kn] G. Knieper, The uniqueness of the measure of maximal entropy for geodesic flows on rank 1 manifolds, preprint, 1996.

[KT] T. Krüger and S. Troubetzkoy, Markov partitions and shadowing for diffeomorphisms with no zero exponents, preprint, 1997.

[LY] T.-Y. Li and J. Yorke, Ergodic transformations from an interval into itself, Trans. Amer. Math. Soc. 235 (1978), 183-192.

[Li] D. Lind, Perturbations of shifts of finite type, SIAM J. Discrete Math. 2 (1989), 350-365.

[MlT] J. Milnor and W. Thurston, On iterated maps of the interval, in: Dynamical Systems (College Park, Md., 1986-87), Lecture Notes in Math. 1342, Springer, Berlin, 1988, 465-563.

[M1] M. Misiurewicz, Horseshoes for mappings of the interval, Bull. Acad. Polon. Sci. Sér. Sci. Math. Astronom. Phys. 27 (1979), 167-169.

[M2] - Continuity of entropy revisited, in: Dynamical Systems and Applications, World Sci. Ser. Appl. Anal. 4, World Sci., 1995, 495-503.

[MS1] M. Misiurewicz and W. Szlenk, Entropy of piecewise monotone mappings, Astérisque 50 (1977), 299-310.

[MS2] - - - Entropy of piecewise monotone mappings, Studia Math. 67 (1980), 45-63.

[P] Ya. Pesin, Characteristic exponents and smooth ergodic theory, Russian Math. Surveys 32 (1977), 55-114.

[Pu] C. C. Pugh, The $C^{1+\alpha}$ hypothesis in Pesin theory, Publ. Math. IHES 59 (1984), $143-161$.

[Q] A. Quas, Invariant densities for $C^{1}$ maps, Studia Math. 120 (1996), 83-88.

Department of Mathematics

The Pennsylvania State University

University Park, Pennsylvania 16802

U.S.A.

E-mail: katok_a@math.psu.edu mezhirov@math.psu.edu 